\section{Cognitive remediation therapy in schizophrenia}

\author{
Randomised controlled trial \\ TIL WYKES, CLARE REEDER, SABINE LANDAU, BRIAN EVERITT, \\ MARTIN KNAPP, ANITA PATEL and RENEE ROMEO
}

\begin{abstract}
Background Cognitive difficulties are prevalent in people with a diagnosis of schizophrenia and are associated with poor long-term functioning.
\end{abstract}

\begin{abstract}
Aims To evaluate the effectiveness of cognitive remediation therapy on cognitive difficulties experienced by people with schizophrenia.
\end{abstract}

Method Participants with a diagnosis of schizophrenia, a social behaviour problem and a cognitive difficulty $(n=85)$ were randomised to 40 sessions of cognitive remediation or treatment as usual in a single-blind randomised controlled trial. Working memory, cognitive flexibility and planning, were measured at weeks 0,14 and 40 .

\section{Results There were durable improvements in working memory (advantage 1.33 points, 95\% $\mathrm{Cl} 0.43-2.16$, standardised effect size 0.34 ) as well as an indication of improvement in cognitive flexibility. Memory improvement predicted improvement in social functioning. Costs were lower in the cognitive remediation group following therapy but rose at follow-up. However, cost-effectiveness analyses showed that improvements in memory were achieved at little additional cost.}

Conclusions Cognitive remediation therapy is associated with durable improvements in memory, which in turn are associated with social functioning improvements in people with severe mental illness.

Declaration of interest None.
Both longitudinal and cross-sectional studies of patients with a diagnosis of schizophrenia suggest that cognitive performance is poor and remains poor over the course of the disorder, and that such deficits, particularly in memory, limit functioning outcomes and the rehabilitation of particular life skills such as work and social functioning (Green et al, 2000; Wykes \& Reeder, 2005). In order to remove this rate limitation a new rehabilitation technology, cognitive remediation therapy, was developed with the aim of improving cognition and thereby increasing the likelihood of improved functioning outcomes. Cognitive remediation therapy is an umbrella term for a number of different interventions defined by their procedural characteristics such as use of a therapist, use of a computer and the method of training. There is some evidence of efficacy for face-to-face therapy from small studies; however, no large study has investigated the effects and cost-effectiveness of face-to-face therapy. In addition, studies have been limited to people who fulfilled narrow entry criteria in terms of their cognitive difficulties. It is, therefore, not yet possible to identify whether this form of cognitive therapy will have an impact on those with a spectrum of cognitive difficulties. The key effectiveness questions for cognitive remediation therapy concern its likely success when the recipients have a variety of cognitive difficulties as well as a diagnosis of schizophrenia, and whether any cognitive improvements have an impact on functioning.

\section{METHOD}

\section{Study design}

We carried out a single-blind, randomised controlled trial of a new therapy to improve cognition in people with schizophrenia by comparing a group receiving 40 sessions of therapy with a group who received only usual treatment. We tested whether cognitive skills improved in the intervention group and whether this improved cognitive skill led to improvements in symptoms, social functioning and self-esteem. After baseline assessment, participants were randomised to either treatment or control and were then assessed at 14 weeks (posttherapy) and 40 weeks (6 months after therapy discontinuation). The trial registration number is ISRCTN44277627.

\section{Inclusion and exclusion criteria}

We recruited participants from local community mental health teams in the South London and Maudsley National Health Service Trust in a structured geographical rotation from February 1999 to December 2002. Patients were included if they had been in contact with the services for at least 1 year, were at least 17 years old, had a diagnosis of schizophrenia based on DSM-IV (American Psychiatric Association, 1994) and evidence of both social functioning, defined as a problem on the Social Behaviour Scale (SBS; Wykes \& Sturt, 1986), and thinking difficulties. Thinking difficulties were defined as a poor memory score on the Rivermead scale (Wilson et al, 1999), and/or cognitive flexibility on the Wisconsin Card Sorting Test (WCST; Heaton et al, 1993) below the 16 th centile, and/or a poor score on the Hayling Sentence Completion Test (Burgess \& Shallice, 1996).

\section{Therapy}

Several programmes are available to test, but the one chosen was first developed in Australia (Delahunty \& Morice, 1993) and incorporates the teaching of strategic information processing, which has been identified as the training more likely to produce larger cognitive benefits following analysis in the most comprehensive review (Krabbendam \& Aleman, 2003). This is a promising programme because, unlike the others, it has been shown to have specific effects when tested in a randomised controlled trial against another psychosocial programme (Wykes et al, 1999, 2003).

Therapy consisted of 40 face-to-face sessions, each involving a number of paper and pencil tasks that provide practice in a variety of cognitive skills that are set out in a manual (Delahunty et al, 2002). Therapy was delivered to individuals on at least 3 days per week until 40 sessions were completed. The therapists were graduate psychologists who had followed a dedicated training programme involving theory, 
observation and supervised practice sessions. The therapy is based on three general clinical principles:

(a) teaching (or facilitating learning of) new efficient information processing strategies;

(b) individualising therapy;

(c) aiding the transfer of cognitive gains into the real world.

The programme consists of three modules: cognitive flexibility, working memory and planning (Delahunty et al, 2002; Reeder et al, 2004). In each module there is a series of tasks, graded from 'extremely easy' to 'easy', so that an errorless learning environment can be provided. In the cognitive flexibility module, patients are given practice in engagement, disengagement and re-engagement activities for a particular cognitive set or between two sets. The working memory module requires the person to maintain two sets of information simultaneously and to carry out transformations on a held information set. The planning module consists of tasks in which the participant has to plan a sequence of moves to acquire a goal. The emphasis in this module is to organise information and to create and use sub-goals. One major change from the therapy as administered in previous studies (e.g. Wykes et al, 1999) was the emphasis of therapists on the possible uses of the strategies being taught within the participants' own lives, for example in going shopping. This was achieved by encouraging the participants to reflect on how the skills learnt in therapy might be used to achieve real-life goals (see Wykes \& Reeder, 2005 for further details).

Therapist fidelity was checked against the records completed at the end of each session, the task sheets produced during the sessions and by direct observation. Participants did receive therapy that complied with the manual, and the majority of the tasks were delivered for most participants. These high levels of fidelity were maintained and supported by weekly supervision.

\section{Outcome measures}

The three main outcome measures were:

(a) cognitive flexibility - categories achieved from the Wisconsin Card Sorting Test;

(b) planning - the profile score from the Behavioural Assessment of the Dysexecutive Syndrome (BADS; Wilson et al, 1996); (c) working memory - total raw score on the Digit Span test of the Wechsler Adult Intelligence Scale III (WAIS-III; Wechsler, 1981).

In addition to the main outcomes we also collected data on symptoms from the Positive and Negative Syndrome Scale (PANSS; Kay et al, 1987), a self-esteem score from the Rosenberg Self-Esteem Scale (SES; Rosenberg, 1965) and level of social functioning from the Social Behaviour Scale (Wykes \& Sturt, 1986). Health, social care and criminal justice system resource use were assessed using the Client Service Receipt Inventory (Beecham \& Knapp, 1992) retrospectively from healthcare staff or records, and/or by participant self-report for the relevant assessment intervals. Unit costs (at 2000-2001 levels) based on national statistics were attached to all resource use to calculate total health and societal costs.

\section{Procedure}

All participants gave written informed consent prior to inclusion in the trial. After baseline assessment participants were randomly allocated by an independent statistician using a concealed randomisation method. Participants assigned to the cognitive remediation condition received therapy within 2 weeks of randomisation. Therapy continued for 40 sessions (approximately 12 weeks). In addition to the assessments on outcome measures, data were also collected on clinical history, demographic characteristics and premorbid IQ as assessed on the National Adult Reading Test (NART; Nelson \& Willison, 1991).

\section{Protecting against bias}

Symptoms were rated by a psychiatrist unaware of group allocation, who was based in a different building to the other researchers and the independent site of randomisation. Participants were informed that they should not reveal their group allocation prior to each assessment and none did so for the symptom assessment. Cognitive data were collected by independent assessors who, although initially masked to group allocation, were not unaware of all allocations since some participants revealed their randomisation group at the post-treatment assessment point. However, as these data were collected either by computer or under clear guidance and instruction, the effect of the revealing of group allocation is unlikely to be significant. Social behaviour data were collected from keyworker or relative informants who were independent of the trial but not masked to group allocation.

\section{Sample size and power of the study}

Previous studies of this programme have suggested that there will be improvement in both groups with repeated testing over time. We have therefore used the outcome data reported by Wykes et al (1999) to define a clinically significant difference as $71 \%$ of the experimental group improving compared with $31 \%$ of the control group. This difference is considered to be a clinically significant difference in proportions considering the amount of therapy time that would need to be allocated. This is an odds ratio of 0.184 . We estimated that a sample of 29 people per group would have $80 \%$ power at the $5 \%$ significance level to detect this difference. The sample size was increased to 42 to take into account a possible $30 \%$ withdrawal rate.

\section{Statistical analyses}

Participants were analysed in the treatment group to which they were randomised irrespective of whether they adhered to their treatment. All outcome measures were analysed using linear mixed modelling with models fitted using restricted maximum likelihood methods based on the assumption of normality for the error terms. Models included baseline values of the outcome measure, and symptoms considered possibly to affect cognitive outcome following therapy as explanatory variables. The experimental factors, randomisation group (therapy or control) and time (posttreatment or follow-up) were included in the model as fixed main effects and a group $\times$ time interaction. In addition, random effects for participants were included. A significant interaction term implies a differential intervention effect at the two posttherapy time points. Where the interaction effect was not significant, the corresponding model was refitted excluding this term, to assess the overall group effect. A main effect of randomisation group would then be interpreted as an effect of the intervention therapy consistent across both time points.

We also chose to investigate whether the effects of treatment meant that cognitive scores were then within the normal range. This differential improvement rate was tested by chi-squared tests for each 
main outcome measure, by investigating the changes to normal scores of all those who had abnormal scores at baseline.

\section{Costs}

Differences in mean costs and $95 \%$ confidence intervals were obtained using nonparametric bootstrapping techniques to account for any non-normality in their distribution (1000 repetitions), using Stata version 8.0 for Windows. Costs (including the costs of therapy where applicable) were adjusted for baseline values of equivalent cost categories and baseline total PANSS score.

To allow a cost-effectiveness analysis based on a more meaningful interpretation of the primary outcome measure, we also compared the percentage of 'improvers' in each group based on WAIS-III Digit Span raw scores (improvers were defined as those gaining 2 points or more on this measure since baseline). This was based on a relatively large effect size of 0.7 and was chosen because recent studies suggest that improvements of this size may contribute to functional improvements (Bryson \& Bell, 2003). For cost-effectiveness ratios based on 'improvers', percentages were also compared using non-parametric bootstrapping, and were adjusted for baseline WAIS-III Digit Span raw score and total PANSS score.

It was not necessary to calculate ratios in scenarios in which one group had both lower costs and better outcomes, as the decision regarding which treatment is preferred is intuitively clear. Where one group had both higher costs and better outcomes, the additional cost per additional $1 \%$ of improvers on the WAIS-III Digit Span raw score was calculated by dividing the mean difference in costs by the mean difference in percentage of improvers.

\section{Mechanisms underlying social functioning change}

Finally, cognitive change is predicted to have an impact on social functioning. In order to test this model a regression was carried out with follow-up social functioning outcome as the dependent variable, therapy group as a factor, and cognitive change over the treatment period and baseline levels of social functioning and symptoms as covariates. The model first tested a group-dependent cognition effect by means of an interaction between cognitive change and group. If the interaction effect was not significant then it was excluded and the model rerun to assess the overall effect of cognitive change on functioning.

\section{RESULTS}

Eighty-five participants were recruited to the trial, of whom 43 were randomised to cognitive remediation therapy and 42 to the control condition (Fig. 1). Nearly three-quarters of the sample were men $(73 \% ; n=62)$ and the mean age was 36 years; $47 \%(n=40)$ were living in independent accommodation or with their family. Most had no experience of a stable relationship, and $40 \% \quad(n=34)$ had never lived independently. About half $(n=44)$ had been in touch with the psychiatric services for at least 10 years. The participants were therefore severely impaired in overall functioning, although some people had made some achievements such as marrying or having independent living arrangements.

Not all participants agreed to complete all assessments for a variety of reasons, including delusional ideation as well as refusal. There was no difference between the groups in the rate of withdrawals $\left(\chi^{2}=0.047\right.$, d.f. $\left.=1, P=1.0\right)$ and none of the potential baseline variables (cognition outcomes, self-esteem, social behaviour or

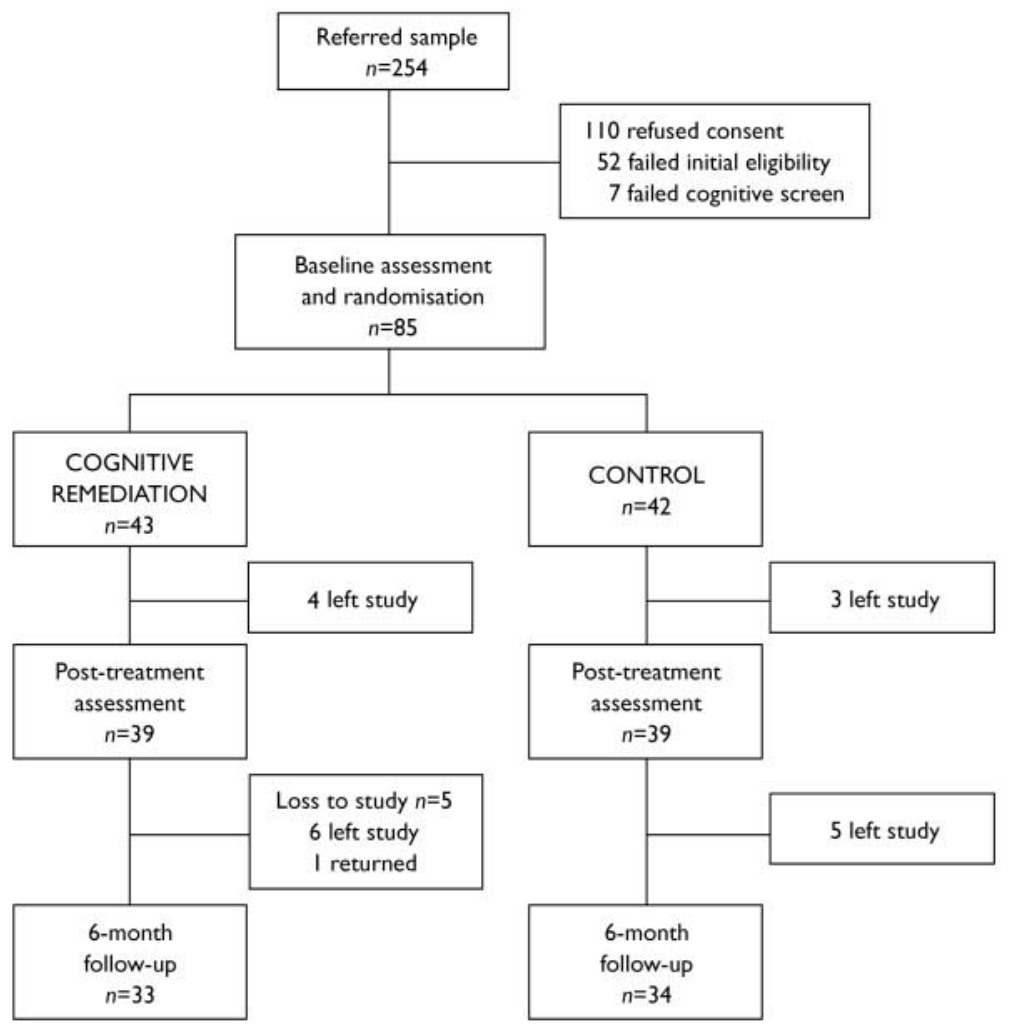

Fig. I Study profile. symptoms) predicted withdrawal from the study (probability levels all above 0.16 ). Overall, the intervention group participants received a mean of $36.9(0-40)$ sessions of therapy, with a mean of 3.8 per week for those who started therapy, and at least 30 sessions being received by $93 \%$ of the sample.

Table 1 shows the types of primary medications and the mean dosage in chlorpromazine equivalents for those whose primary medication was a typical antipsychotic agent. Two people in the therapy group and one person in the control group received both typical and atypical medication. Of those prescribed typical antipsychotics, 11 received them in the form of depot preparations ( 4 in the therapy group and 7 in the control group).

As would be expected after random treatment allocation, the main cognitive outcomes were similar in the two groups at baseline, as were social behaviour and self-esteem. However, despite randomisation, the level of symptoms appeared to be greater in the therapy group (Table 2), but this variable was already included as a covariate in all models considered. The baseline means for the other main and secondary outcomes are presented in Table 2. The NART scores were 92.7 (s.d.=13.3) for the therapy group and 92.4 (s.d.= 12.7) for the control group. 


\section{Outcomes of therapy}

Table 3 shows the group comparison results for all the outcomes. All statistically significant changes show an advantage for cognitive remediation. Working memory shows an improvement across both posttreatment time points and cognitive flexibility an improvement at the followup time point. Both differences are small to moderate effects. For working memory change the number needed to treat (NNT) is 3.1 to produce a clinical change of at least 2 points on the Digit Span test. For cognitive flexibility the NNT is 6.7 to improve by at least two categories on the WCST at follow-up.

Using the same mixed models analyses, drug effects were investigated using drug type as an additional explanatory variable. Neither working memory nor WCST outcomes were related to the type of medication prescribed (defined as either typical $v$. atypical or when depot preparations were considered). However, there was a significant drug $\times$ group interaction $\left(F_{(2,70)}=4.4\right.$, $P=0.016)$ for planning scores. Further investigation of these effects suggests that cognitive remediation therapy had an overall effect on planning for those who received either clozapine or typical medication that was absent for those who received other atypical medications.

\section{Normal score attainment following therapy}

The normal range for the main outcome tests was conservatively estimated from

Table I Antipsychotic medication provided at baseline

\begin{tabular}{lcc}
\hline & $\begin{array}{c}\text { Therapy group } \\
(n=43)\end{array}$ & $\begin{array}{c}\text { Control group } \\
(n=42)\end{array}$ \\
\hline Atypical medication, $n$ & & 12 \\
Clozapine & 16 & 12 \\
Olanzapine & 8 & 2 \\
Risperidone & 7 & 2 \\
Amisulpride & 1 & 1 \\
Quetiapine & 2 & 300 \\
Typical medication & & \\
Mean dosage, mg CPZeq & 368 & 2 \\
\hline
\end{tabular}

CPZeq, chlorpromazine equivalent.

the test manuals. For working memory this was within 1 standard deviation of the mean normal score, for cognitive flexibility it was above the 5th percentile and for planning it was above the low average score.

For working memory there were 21 participants in the therapy group and 18 in the control group who had abnormal working memory scores at baseline. Following the intervention, there was an advantage to therapy which was significant at the post-therapy assessment but failed to reach significance at follow-up (posttreatment: 43 therapy $v .11 \%$ control, Fisher's exact test $P=0.037$; follow-up: 32 therapy $v .7 \%$ control, Fisher's exact test $P=0.10$ ). For cognitive flexibility there was no difference at either the posttreatment or the follow-up assessment (post-treatment: 15 therapy $v . \quad 17 \%$ control; follow-up: 17 therapy $v .21 \%$ control). For planning, although almost double the number of people in the therapy group had a normalised score, there was no statistically significant effect (posttreatment: 32 therapy $v .17 \%$ control; follow-up: 36 therapy $v .19 \%$ control).

\section{Other outcomes}

These analyses are designed to detect whether there is a direct effect of cognitive remediation therapy on functioning outcomes, irrespective of the level of cognitive improvement detected. The results of the analyses are shown in Table 3. For both symptoms and self-esteem the results were in the expected direction, with the therapy group improving compared with the control group at post-treatment. There was evidence of an interaction such that any differential improvement at the

Table 2 Cognitive, secondary and functioning outcome scores

\begin{tabular}{|c|c|c|c|c|c|c|c|c|c|c|c|c|}
\hline & \multicolumn{4}{|c|}{ Baseline assessment } & \multicolumn{4}{|c|}{ Post-treatment assessment } & \multicolumn{4}{|c|}{ Follow-up assessment } \\
\hline & \multicolumn{2}{|c|}{ CRT group } & \multicolumn{2}{|c|}{ Controls } & \multicolumn{2}{|c|}{ CRT group } & \multicolumn{2}{|c|}{ Controls } & \multicolumn{2}{|c|}{ CRT group } & \multicolumn{2}{|c|}{ Controls } \\
\hline & $n$ & Mean (s.d.) & $n$ & Mean (s.d.) & $n$ & Mean (s.d.) & $n$ & Mean (s.d.) & $n$ & Mean (s.d.) & $n$ & Mean (s.d.) \\
\hline Memory (Digit Span)' & 43 & $14.2(3.9)$ & 42 & I5.I (3.9) & 39 & $15.5(3.3)$ & 38 & $14.8(3.3)$ & 36 & I5.I (3.7) & 34 & $14.6(4.1)$ \\
\hline Cognitive flexibility (WCST) ${ }^{2}$ & 43 & $2.4(1.5)$ & 42 & $2.2(1.3)$ & 37 & $2.3(2.4)$ & 37 & $2.4(2.2)$ & 35 & $2.8(2.2)$ & 31 & $2.3(2.4)$ \\
\hline Planning (BADS) ${ }^{3}$ & 43 & II.7 (4.6) & 42 & $12.7(5.1)$ & 38 & I3.5 (4.8) & 38 & I3.5 (4.6) & 35 & I3.6 (4.3) & 34 & I3.3 (5.6) \\
\hline \multicolumn{13}{|l|}{ Symptoms (PANSS) } \\
\hline Total score & 43 & $62.9(16.4)$ & 42 & $56.9(14.7)$ & $4 I$ & $60.1(18.6)$ & 39 & $59.8(17.5)$ & 36 & $61.3(20.1)$ & 35 & $56.2(13.6)$ \\
\hline Negative symptoms & 43 & $18.6(17.3)$ & 42 & $16.6(7.2)$ & 41 & $17.0(16.6)$ & 39 & $16.6(7.3)$ & 36 & $17.2(7.4)$ & 35 & $15.9(6.8)$ \\
\hline Positive symptoms & 43 & $14.2(5.4)$ & 42 & $12.5(5.0)$ & 41 & $14.2(5.4)$ & 39 & $13.8(6.4)$ & 36 & $14.6(6.4)$ & 35 & $12.8(4.7)$ \\
\hline Self-esteem (SES) & 43 & $17.3(4.4)$ & 42 & $16.7(4.2)$ & 38 & $17.9(4.2)$ & 39 & $16.6(4.0)$ & 35 & $17.7(4.0)$ & 34 & $17.7(3.2)$ \\
\hline Social behaviour (SBS) & 43 & II.6(8.5) & 42 & I3.7 (II.2) & $4 I$ & $10.0(7.6)$ & 38 & II.3 (7.3) & 41 & $11.6(8.7)$ & 35 & II.3 (8.3) \\
\hline
\end{tabular}

BADS, Behavioural Assessment of the Dysexecutive Syndrome; CRT, cognitive remediation therapy; PANSS, Positive and Negative Syndrome Scale; SES, Self-Esteem Scale; WCST, Wisconsin Card Sorting Test; SBS, Social Behaviour Scale.

I. Maximum score 30.

2. Maximum score 6 .

3. Maximum score 24 . 


\begin{tabular}{|c|c|c|c|c|}
\hline Outcome measures & Interaction & $\begin{array}{c}\text { Group effect } \\
\text { (excluding non-significant } \\
\text { interaction) }\end{array}$ & $\begin{array}{l}\text { Estimated advantage to } \\
\qquad \text { CRT' }^{(95 \% \mathrm{Cl})}\end{array}$ & $\begin{array}{l}\text { Standardised } \\
\text { effect size } \\
(95 \% \mathrm{Cl})\end{array}$ \\
\hline \multicolumn{5}{|l|}{ Primary outcomes } \\
\hline Working memory (Digit Span) & $F_{(1,72)}=0.21, P=0.84$ & $F_{(1,71)}=5.82, P=0.019$ & $1.33(0.43$ to 2.16$)$ & $0.34(0.1$ to 0.55$)$ \\
\hline Cognitive flexibility (WCST) & $F_{(1,61)}=6.924, P=0.011$ & NA & & \\
\hline Post-treatment & & & $0.17(-0.64$ to 0.98$)$ & \\
\hline Follow-up & & & I (0.17 to 8.0$)$ & $0.47(0.08$ to 3.77$)$ \\
\hline Planning (BADS) & $F_{(1,67)}=0.315, P=0.576$ & $F_{(1,72)}=2.9, P=0.092$ & I.I ( -0.18 to 2.4$)$ & \\
\hline \multicolumn{5}{|l|}{ Secondary outcomes } \\
\hline Self-esteem (SES) & $F_{(1,71)}=4.44, P=0.039$ & NA & & \\
\hline Post- treatment & & & $1.05(-0.3$ to 2.42$)$ & \\
\hline Symptoms (PANSS) & $F_{(1,72)}=3.55, P=0.06$ & NA & & \\
\hline Post-treatment & & & $-4.68(-10.81$ to 1.44$)$ & \\
\hline Follow-up & & & $1.59(-4.8$ to 8.04$)$ & \\
\hline Social functioning & $F_{(1,76)}=0.438, P=0.51$ & $F_{(1,74)}=0.008, P=0.929$ & & \\
\hline
\end{tabular}

BADS, Behavioural Assessment of Dysexecutive Syndrome; CRT, cognitive remediation therapy; NA, not applicable; PANSS, Positive and Negative Syndrome Scale; SES, Self-Esteem Scale; WCST, Wisconsin Card Sorting Test.

I. Number of points on outcome measure scale.

2. Effect/baseline s.d.

post-treatment assessment disappeared at follow-up. However, there was no evidence of a direct effect of therapy on social behaviour scores.

\section{Economic outcomes}

Total overall health and societal costs are shown in Table 4. There is an advantage (although with highly skewed confidence limits) for the treatment period, with a difference of UK $£ 1086$ in healthcare costs and $£ 1284$ in societal costs in favour of the therapy group, but costs are higher at follow-up. The intervention dominates at the post-treatment evaluation with both lower costs and a greater proportion of participants showing cognitive improvement (mean difference $21 \%, 95 \%$ CI $0-41$ ). At follow-up, the cognitive advantage of the intervention therapy (mean difference $21 \%, 95 \%$ CI 2-41) needs to be considered against the additional costs. The costeffectiveness ratios showed that for each additional $1 \%$ of 'improvers' in Digit Span (see Method) there was an additional cost of $£ 46$ in healthcare and social care costs and $£ 24$ in societal costs.

\section{Effects of cognitive improvement}

Since group could be shown to affect working memory, the effect of working memory change over the treatment period on social functioning outcome to follow-up was investigated using regression. There was no evidence of a group-dependent effect of cognition $(F=0.996$, d.f. $=1.65, P=0.32)$, but after excluding the interaction term there was a significant effect of cognitive change $(F=4.78$, d.f. $=1,66, P=0.03)$, suggesting that improvements in cognition were associated with improvements in social behaviour. When the effects were investigated in each group independently there was a significant effect for the therapy group $\left(F_{(1,35)}=9.2, P=0.005\right)$ but not for the control group $\left(F_{(1,34)}=0.06, P=0.16\right)$.

\section{DISCUSSION}

The participants in this study had a wider range of abilities than participants in other studies and most other clinical information showed a wide spread of scores. In the overall comparisons these wider ranges tended to produce differences in the direction of this sample having poorer performance (e.g. on the WCST and Digit Span). In fact, although the sample was recruited as having fulfilled a number of different cognitive criteria, a large proportion (30 v. 6\%) did not complete any categories

Table 4 Baseline-adjusted mean costs and mean cost differences, including the cost of CRT

\begin{tabular}{|c|c|c|c|c|c|}
\hline & \multicolumn{2}{|r|}{ CRT } & \multicolumn{2}{|r|}{ Usual care } & Mean difference' \\
\hline & $n$ & $\begin{array}{c}\text { Cost, } £ \text { Mean } \\
\text { (s.d.) }\end{array}$ & $n$ & $\begin{array}{c}\text { Cost, } £ \text { Mean } \\
\text { (s.d.) }\end{array}$ & $(95 \% \mathrm{Cl})$ \\
\hline \multicolumn{6}{|l|}{ Post-treatment } \\
\hline Health/social care costs & 41 & $7756(5936)$ & 39 & 8271 (7494) & $-1086(-3146$ to II52) \\
\hline Societal costs & 41 & $8868(5849)$ & 39 & $9497(7413)$ & $-1284(-3348$ to 942$)$ \\
\hline \multicolumn{6}{|l|}{ Follow-up } \\
\hline Health/social care costs & 41 & $15639(12453)$ & 37 & $13426(12852)$ & $975(-3330$ to 5255$)$ \\
\hline Societal costs & 41 & 17586 (12 197) & 37 & $15735(12654)$ & $494(-3564$ to 4577$)$ \\
\hline
\end{tabular}

CRT, cognitive remediation therapy.

I. Adjusted for the baseline values of equivalent cost categories and baseline total Positive and Negative Syndrome Scale score. 
of the WCST unlike participants in an earlier study (Wykes et al, 1999). The current group had also been in contact with psychiatric services for slightly longer and had poorer cognitive capacity than the previous study group (NART IQ $93 v$. 104). Thus the sampling method achieved a wider variation in scores, with more people who had particularly poor cognitive performance on the outcome measures.

Despite the more chronic nature of impaired functioning in this sample, the results were similar to those of the previous pilot study. There was a durable improvement in working memory 6 months after the end of therapy, a significant improvement at follow-up in cognitive flexibility, and an advantage - but not a significant one - for planning (before medication was taken into account). Thus, for the primary cognitive outcomes there is evidence of overall effectiveness in a mixed group of participants, which in itself is an achievement as all the data point to a stable course for cognitive deficits. This fits into the growing evidence base showing that improvements in cognition are achievable even when the disorder has been evident for some time. In fact, nearly half of the people who scored at a very poor level on working memory performed within the normal range following therapy.

Following the end of therapy there was a continuing improvement in cognition, in cognitive flexibility. One possible explanation is that cognitive remediation therapy 'jump starts' engagement in the cognitive system through enhancing positive reward. This is achieved by the reinforcing nature of the tasks; the encouragement within therapy to engage these cognitive systems in everyday tasks; and the improved self-esteem and self-efficacy that further encourage engagement in new tasks, which provides continued practice.

The type of medication prescribed did not have any effect on the WCST or Digit Span outcomes. However, for the planning measure there was a significant group by medication interaction, suggesting that for some cognitive outcomes the type of medication could hinder or enhance the effects of cognitive remediation. The participants prescribed clozapine had a lower baseline planning score and therefore more chance for improvement. On the other hand, there was no difference in cognitive outcome between those taking typical and those taking atypical antipsychotics, suggesting that atypical medication does have a detrimental effect on the likelihood of change due to cognitive remediation.

In addition to cognitive outcomes, there were also some notable improvements in distal outcomes such as self-esteem posttreatment. These effects fit into the growing pattern (described by Twamley et al, 2003) of an effect on functioning of successful cognitive rehabilitation. There was an advantage in terms of both healthcare and societal costs for the therapy group at the post-treatment assessment, although this was not significant.

\section{Methodological considerations}

Data from other studies demonstrate high levels of stability of cognitive functioning without an intervention (Greig et al, 2004) and it might be proposed that these cognitive effects result only from attention and increased social contact. However, they are similar to those found in a previous trial in which there was an attention control group (Wykes et al, 1999, 2003), and although levels of social contact were higher in the therapy group there was no direct effect on social functioning outcomes. It therefore seems parsimonious to assume that the intervention therapy did produce the beneficial cognitive effects.

It is also vital to consider any possible challenges to the study validity, which for studies of psychological interventions in particular include rater bias. Although procedures were in place to reduce the chance of unmasking group allocation this did happen in some cases when cognitive data were collected. However, data quality checks (double scoring, data entry checking, etc.) were carried out each month to ensure that data were not obviously biased for any one rater or for any one participant. It was not possible to mask group allocation for the social functioning informants, but in this case there was no measurable effect of therapy, only interactions with cognitive improvements that were unknown to the key informants when they were providing the relevant information. It therefore seems likely that these acknowledged methodological difficulties did not compromise the study validity.

The sample size was small (although larger than any prior studies of this therapy) and so the power to investigate subtle effects was low. However, if this therapy is to be provided by health services it needs to show at least moderate effects and the current effect sizes are similar to those attributed to cognitive-behavioural therapy (Tarrier \& Wykes, 2004) suggesting that they may have some clinical relevance.

\section{The effects of improving working memory}

Poor memory has been highlighted in a number of studies as predicting poor overall outcome (Mueser et al, 1991; Green et $a l, 2000)$. It was assumed that cognitive improvements would lead to functional change, and this is one of the reasons that cognitive remediation therapy was developed. However, few studies have measured the functional effects at a time when it might be possible for cognitive improvement to have had time to translate into functional changes. In this study there was support for a model in which a change in working memory had a beneficial effect on social behaviour 6 months after the end of therapy. This effect was only significant in the therapy group. The intervention was associated with lower costs at posttreatment assessment, and even when there were higher costs at follow-up these were small in relation to the beneficial outcome in working memory. The therapy itself has been estimated to cost $£ 546.97$ at 20002001 prices per patient (Wykes et al, 2003). The cost-effectiveness analysis has demonstrated that this translates into a small price to pay for memory improvements which are likely to produce further benefits on social behaviour.

The mechanisms through which cognitive change leads to functioning change have been somewhat elusive. Wykes \& Reeder (2005) have suggested that for change to occur in routine behaviours, cognitive capacity of the sort measured by neuropsychological tests must change. This increases the efficiency with which routine cognitive schemas are implemented or held online. However, most functioning is not routine and must be flexible, so for novel behaviours it is necessary to change a further aspect of thinking - metacognition. Metacognitive processes and knowledge are required for the replacement of inefficient existing routine schemas and for the production of new, temporary high-level schemas. Current measures of cognition do not allow the separation of cognitive from metacognitive processes, and current measures of functioning also do not allow the differentiation of routine from novel actions. It is possible that the incomplete translation of working memory improvements into 
social behaviour change is explained by the fact that it is not currently possible to differentiate the two forms of action. Working memory improvements are likely to translate into routine actions immediately, but not necessarily directly to non-routine actions unless metacognition has also improved. Future studies need to be able to differentiate behaviour change into routine behaviour efficiency and novel behaviour flexibility.

\section{ACKNOWLEDGEMENTS}

The study was supported by a grant from the Department of Health (RFG 757) who had no role in the study design, data analysis or interpretation, writing the report or decision to submit the paper for publication. The views expressed in this paper are those of the authors and not necessarily those of the funder.

\section{REFERENCES}

American Psychiatric Association (1994) Diagnostic and Statistical Manual of Mental Disorders (4th edn) (DSM-IV). APA

Beecham, J. K. \& Knapp, M. R. J. (1992) Costing psychiatric interventions. In Measuring Mental Health Needs (eds G. Thornicroft, C. Brewin \& J. K.Wing), pp. 163-183. Gaskell.

Bryson, G. \& Bell, M. D. (2003) Initial and final work performance in schizophrenia: cognitive and symptom predictors. Journal of Nervous and Mental Disease, 191, 87-92.

Burgess, P. \& Shallice, T. (1996) Response suppression initiation and strategy use following frontal lobe lesions. Neuropsychologia, 34, 263-273.

Delahunty, A. \& Morice, R. (1993) A Training Programme for the Remediation of Cognitive Deficits in Schizophrenia. Department of Health of Australia.

TIL WYKES, MPhil, PhD, CLARE REEDER, DClinPsych, PhD, Department of Psychology, SABINE LANDAU, PhD, BRIAN EVERITT, BSc, Department of Biostatistics and Computing; MARTIN KNAPP, PhD, ANITA PATEL, PhD, RENEE ROMEO, PhD, Department of Health Services Research, King's College London, Institute of Psychiatry, London, UK

Correspondence: Professor Til Wykes, Department of Psychology, PO Box 77, Institute of Psychiatry, De Crespigny Park, London SE5 8AF, UK. Tel: +44 (0) 0207848 0596; fax: +44 (0) 02078485006 ; email: t.wykes@iop.kcl.ac.uk

(First received 16 May 2006, final revision 30 November 2006, accepted 5 January 2007)

Delahunty, A., Reeder, C., Wykes, T., et al (2002) Revised Cognitive Remediation Therapy Manual. Institute of Psychiatry, London.

Green, M. F., Kern, R. S., Braff, D. L., et al (2000) Neurocognitive deficits and functional outcome in schizophrenia: are we measuring the 'right stuff'? Schizophrenia Bulletin, 26, 119-136.

Greig, T., Nicholls, S., Wexler, B., et al (2004) Testretest stability of neuropsychological testing and individual differences in variability in schizophrenia outpatients. Psychiatry Research, 129, 24I-247.

Heaton, R. K., Chelune, G. J., Talley, J. L., et al (1993) Wisconsin Card Sorting Test Manual (revised and expanded). Psychological Resources.

Kay, S. R., Fizbein, A. \& Opler, L. A. (1987) The Positive and Negative Syndrome Scale (PANSS) for schizophrenia. Schizophrenia Bulletin, 13, 261-276.

Krabbendam, L. \& Aleman, A. (2003) Cognitive rehabilitation in schizophrenia: a qualitative analysis of controlled studies. Psychopharmacology, 169, 376-382.

Mueser, K.T., Bellack, A. S., Douglas, M. S. et al (1991) Prediction of social skill acquisition in schizophrenic and major affective-disorder patients from memory and symptomatology. Psychiatry Research, 37, $281-296$

Nelson, H. E. \& Willison, J. R. (199I) National Adult Reading Test (NART): Test Manual (2nd edn). nferNelson.

Reeder, C., Newton, E., Frangou, S., et al (2004)

Which executive skills should we target to affect social functioning and symptom change? A study of a cognitive remediation therapy program. Schizophrenia Bulletin, 30 $87-100$
Rosenberg, M. (1965) Society and the Adolescent Selfimage. Princeton University Press.

Tarrier, N. \& Wykes, T. (2004) is there evidence that cognitive behaviour therapy is an effective treatment for schizophrenia? A cautious or cautionary tale? Behaviour Research and Therapy, 42, 1377-1401.

Twamley, E. W., Jeste, D. V. \& Bellack, A. S. (2003) A review of cognitive training in schizophrenia. Schizophrenia Bulletin, 29, 358-382.

Wechsler, D. (198I) Wechsler Adult Intelligence Scale: Revised. Harcourt.

Wilson, B., Alderman, N., Burgess, P., et al (1996) Behavioural Assessment of the Dysexecutive Syndrome BADS. Thames Valley Test Company.

Wilson, B., Clare, L., Cockburn, J., et al (1999)

Rivermead Behavioural Memory Test. Thames Valley Test Company.

Wykes, T. \& Reeder, C. (2005) Cognitive Remediation Therapy for Schizophrenia. Routledge.

Wykes, T. \& Sturt, E. (1986) The measurement of social behaviour in psychiatric patients: an assessment of the reliability and validity of the SBS schedule. British Journal of Psychiatry, 148, I-11.

Wykes, T., Reeder, C., Corner, J., et al (1999) The effects of neurocognitive remediation on executive processing in patients with schizophrenia. Schizophrenia Bulletin, 16, 199-207.

Wykes, T., Reeder, C., Corner, J., et al (2003) Are the effects of cognitive remediation therapy (CRT) durable? Results from an exploratory trial in schizophrenia. Schizophrenia Research, 61, 163-174. 